
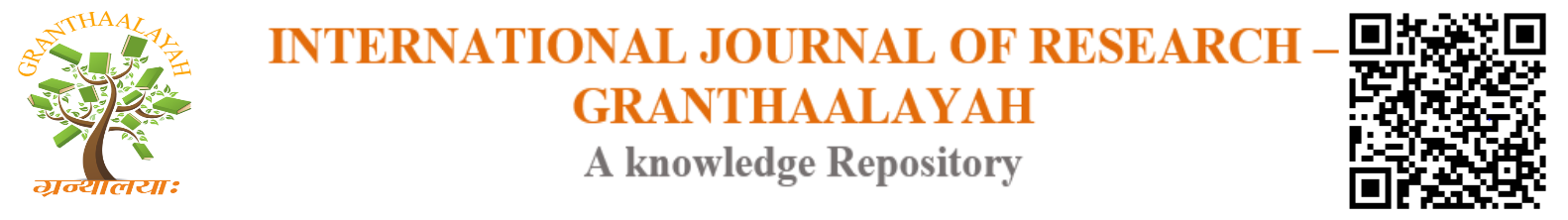

Social

\title{
RAIPUR PUBLISHED IN NEWSPAPERS RELATED TO SECULARISM STUDY REPORTS
}

(Economic, Dainik Bhaskar, with particular reference to the New World and the magazine in Hindi daily newspaper)

रायपुर से प्रकाशित समाचार पत्रों में धर्म-निरपेक्षता से सम्बंधित खबरों का अध्ययन (नवभारत, दैनिक भास्कर, नई दुनिया और पत्रिका हिन्दी दैनिक न्यूज पेपर के विशेष संदर्भ में)

\author{
Chandrashekhar Prasad ${ }^{* 1}$, Dr. Rajendra Mohanty ${ }^{2}$ \\ ${ }^{*}$ MPhil. Researcher, Media Studies, Journalism and Mass Communication, Kushabhau Thakre \\ University, Raipur (Chhattisgarh), INDIA \\ ${ }^{2}$ Assistant Professor, Department of Head Media Studies, Journalism and Mass Communication, \\ Kushabhau Thakre University, Raipur (Chhattisgarh), INDIA
}

DOI: https://doi.org/10.29121/granthaalayah.v4.i6.2016.2649

\section{ABSTRACT}

As announced in the preamble of the Constitution, India is a secular country. This is a particular religion, race, religion, caste, sex or place of birth is discrimination against members. Amendment 42 in the preamble word secular in 1976, was inserted by 2. The equality of all religions and religious tolerance and respect means. Raipur is the capital of the Chhattisgarh Ayedin change is seen in the city since. The city is home to all religions came from across the country. As well as numerous reports related reports also found secularism. Published today by the Economic Raipur, Bhaskar, magazine and news related to the New World on the secularism is working. It is true that such reports are published is bound to be a change in people's thinking. Changes in society, such reports cannot be ranked as the people of the opinion that such reports should prominently place. Also, given the severity of the news stories related pages, photos etc. is determined and how much information is to take. Raipur related to research in the field of study has been published in newspapers selected. Economic researchers said, Bhaskar, and the new world of news magazine studied 15 to August 27. Submitted analytical research technique has been used for research. Which in this context the importance of newspaper reports linked it, it also has been studied. With the news that the focus is on photos, the photo was taken or not. So what if I've found the location. After appearances amid reports of how the readers are Pratikria etc are analyzed.

Keywords:

published newspaper, secularism study, related pages. 


\section{सारांश}

भारतीय संविधान के प्रस्तावना में घोषणा के अनुसार, भारत एक धर्म-निरपेक्ष देश है। यह एक खास धर्म, जाति, धर्म, जाति, जन्म की सेक्स या जगह के सदस्यों के खिलाफ भेदभाव है। अवधि धर्मनिरपेक्षता के लिए भारतीय धारणा अवधि के लिए फ्रेंच राष्ट्र से अलग है। धर्मनिरपेक्ष शब्द प्रस्तावना में ४२ संशोधन, 1976, 2 द्वारा डाला गया था। यह सभी धर्मों और धार्मिक सहिष्णुता और सम्मान की समानता का अर्थ है। रायपुर को जब से सत्तीसगढ़ की राजधानी बनाया गया है जब से शहर में आयेदिन परिर्वतन देखने को मिलता है। दूसरी विशेष बात यह है कि इस शहर में मीडिया ने भी पैर बहुत तेजी से पसारा, जिसमें प्रिंट मीडिया सबसे आगे हैं। दैनिक अखबारों के अलावा सांध्य और कॉपैक्ट अखबारों ने अपनी-अपनी जगहें बनायीं। इस शहर में देशभर से आये सभी धर्मों के लोग रहते हैं। तमाम तरह की खबरों के साथ-साथ धर्म-निरपेक्षता से सम्बंधित खबरों का भी स्थान मिला। आज रायपुर से प्रकाशित नवभारत, भास्कर, पत्रिका और नई दुनिया ने धर्म-निरपेक्षता से जुड़ी हुई खबरों पर भी काम कर रहा है। यह सत्य है कि इस तरह की खबरें प्रकाशित होने से लोगों की सोच में बदलाव आना स्वाभाविक है। समाज में बदलाव लाने के लिए इस तरह की खबरों का जितना स्थान मिलना चाहिए नहीं मिलता, जबकि लोगों की राय है कि इस तरह की खबरों का प्रमुख्ता से स्थान देना चाहिए। कभी-कभी बिना फोटो की खबरें छोटी कर दी जाती है जो उचित नहीं है।

Cite This Article: Chandrashekhar Prasad, and Dr. Rajendra Mohanty, "RAIPUR PUBLISHED IN NEWSPAPERS RELATED TO SECULARISM STUDY REPORTS" International Journal of Research - Granthaalayah, Vol. 4, No. 6 (2016): 157-162.

\section{1 प्रस्तावना}

रायपुर छत्तीसगढ़ की राजधानी है। यह देश का 26 वां राज्य है। 1 नवम्बर 2000 के मध्य-प्रदेश से विभाजित छत्तीसगढ़ का निर्माण किया गया। रायपुर खारुन नदी के तट पर बसा छत्तीसगढ़ का सबसे बड़ा शहर है। रायपुर एक बड़े मैदान के मध्य में स्थित है जो धान का कटोरा के नाम से भी जाना जाता है। किसी देश में प्राय किसी धर्मविशेष के मानने वालों का बहुमत रहता है। हिंदुस्तान में हिंदू पाकिस्तान में मुसलमान, इसरायल में यहूदी, बर्मा, श्रीलंका, स्याम आदि में बौद्ध बहुसंख्यक हैं। इसी तरह ब्रिटेन, यूरोप उत्तरी और दक्षिण अमरीका, आस्ट्रेलिया आदि में ईसाई धर्म के अनुयायियों का बहुमत है।

बहुमत के कारण वहां के सांस्कृतिक वातावरण में, वहां के धर्म की छाप लगना स्वाभाविक है। किंतु कोई भी राज्य, राज्य के रूप में, किसी धर्मविशेष से अलग रह सकता है। भारत का वर्तमान संविधान तथा लोकतंत्रीय प्रणाली इसके ज्वलंत उदाहरण है। यही कारण है कि अन्य शहरों के अलावा रायपुर में भी प्रिंट मीडिया में आयेदिन नये-नये प्रयोग हो रहे हैं। नवभारत, भास्कर, नई दुनिया और पत्रिका आदि दजनों अखबरों का प्रकाशन होता है। कुछ वर्ष पहले की बात करे तो खबरों को लेकर अखबारों में एक सीमित सोच थी, लेकिन आज इसका क्षेत्रफल विस्तृत रूप धारण कर लिया है। देखा जाए तो राजनीति, अर्थ व्यस्था, शिक्षा, खेल-कूद, खेती-किसानी, मौसम, महिलाएं, बच्चे, बाजारीकरण और धर्म-निरपेक्षता से जुड़ी हुई आदि खबरों को लेकर रायपुर से प्रकाशित समाचार पत्रों में स्थान दिया जा रहा है। रायपुर से प्रकाशित समाचार पत्रों में धर्म-निरपेक्षता से सम्बंधित खबरों के लिए पेज निर्धारित नहीं है। इसके अलावा इससे जुड़ी हुई खबरों की गंभीरता को देखते हुए पेज, फोटो और कितनी बड़ी खबरें लेनी है आदि निर्धारित किया जाता है। रायपुर के पूर्व में महानदी नदी बहती है। उत्तर-पश्चिम में मैकाल की पहाडियां हैं। उत्तरी ओर छोटा नागपुर का पठार और दक्षिण में बस्तर का पठार है। रायपुर मुम्बई-हावड़ा रेल लाइन 
पर है और यह सभी महत्वपूर्ण शहरों से जुड़ा है। आकाशवाणी का मीडियम वेब पर एक रेडियो स्टेशन 2 अक्टूबर 1974 से कार्य कर रहा है। नई पीढ़ी के लिये एफ एम बैंड पर नए चौनल्स लाये गए। जिनमें विविध भारती या विज्ञापन प्रसारण सेवा 1 जनवरी 2001 को शुरू हुई। तीन नए रेडियो स्टेशन सन् 2007 में शुरू हुए हैं। इनमें रेडियो मिर्ची, रेडियो रंगीला तथा रेडियो माई एफ एम का चौनल है। वर्ष 2009 में बिना किसी औपचारिक उद्घाटन के एक और नया रेडियो चौनल रेडियो तड़का भी आ गया है। सभी का प्रसारण रायपुर से होता है। टेलीविजन चौनलों में 1977 में शुरू हुआ दूरदर्शन का चौनल सर्व प्रथम है। अब तो दूरदर्शन का डीडी न्यूज चौनल भी रायपुर में देखा जा सकता है। सहारा समय, ई टीवी न्यूज, जी छत्तीसगढ़ 24 घंटे, वॉच न्यूज के साथ केबल टीवी केएम चौनल और ग्रैन्ड चौनल है।

\section{2 साहित्य का पुनरावलोकन}

समाचार पत्रों में प्रकाशित धर्म-निरपेक्षता से सम्बंधित खबरों का अध्ययन विषय पर शोध न के बराबर हुए हैं, लेकिन तमाम साहित्यकारों और सम्पादकों ने इस विषय पर प्रकाश डाला जिससे कुछ जूड़ी हुई जानकारियों जरूर मिली जो शोध के लिए मददगार साबित हुई। एक धर्मनिरपेक्ष राज्य धर्मनिरपेक्षता की एक अवधारणा है, जिसके तहत एक राज्य या देश स्वयं को धार्मिक मामलों में आधिकारिक तौर पर, न धर्म और न ही अधर्म का समर्थन करते हुए, तटस्थ घोषित करता है, परंतु भारतीय वाङ्मय में धर्म शब्द का अर्थ अत्यंत व्यापक है।

ज्ञानकोश विकिपीडिया से

1. डॉ. नीतीश ने अपने शोध में जिक्र किया है कि मीडिया का जितना कवरेज हिन्दू धर्म के पर्व-त्योहारों को मिलता है, उतना कवरेज दूसरे धर्मों के पर्व-त्योहारों को शायद ही नसीब होता है। इसके अलावा धर्म-निरपेक्षता से जुड़ी हुई खबरें भी हिन्दू धर्म से जोड़ कर पेश की जाती है।

www.patrakarpraxis.com

2. दूसरे शोध में भी कहा है कि टीवी पर शुरू हुए एक नए ऐतिहासिक कार्यक्रम में जानबूझकर अकबर को एक मुस्लिम आक्रान्ता और खलनायक के रूप में दिखाने की कोशिश की गयी है। यह अकबर जैसे उदारवादी और धर्मनिरपेक्ष शासक का गलत चित्रण कर नयी पीढ़ी को भ्रमित करने की कोशिश है।

3. प्रो. राकेश सिहा ने शोध के द्वारा बताया कि इस देश में जो शांति काल के दंगाई हैं वे वास्तव में धर्म निरपेक्षता और राष्ट्रीय एकता के शत्रु हैं एवं उर्दू अखबारों का एक बड़ा वर्ग उनका वैचारिक जमीन तैयार करता है। जबकि हिन्दी के अखबार कम है।

www.indiapolicyfoundation.org

4. धर्मनिरपेक्षता शब्द को संविधान में न रखने का एक और कारण था धर्म के नाम पर भारत का विभाजन। इतनी बड़ी त्रासदी अगर देश को झेलनी पड़ी तो उसके पीछे भी एक आक्रामक धर्म था। सन् $9 ६ 46,47$ के दंगों ने महात्मा गांधी समेत बहुत से राजनैतिक नेताओं की सोच बदल दी थी। उस दौरान तमाम अखबारों ने भी प्रमुख्ता से स्थान दिया।

मोहनदास करम चन्द्र गांधी -

5. धर्म मानव समभ्यता के प्रारंभ से ही किसी न किसी रूप में मानव जीवन को प्रभावित करता है। धर्म-निरपेक्षता जैसी खबरों का स्थान अखबारों में मिलना चाहिए। धर्म मानव का अपने से परे एक ऐसी 
शक्ति में विश्वास है जिससे वह अपनी संवेगात्मक आवश्यकताओं की संतुष्टि करता है और जीवन में स्थिरता प्राप्त करता है और जिसे वह उपासना व सेवा के माध्यम से अभिव्यक्त करता है।

भारतीय परिप्रेक्ष्य में धर्म-निरपेक्षता - लेखक कृष्ण कुमार यादव

www.srijangatha.com

6. अखबार में जब यह पढ़ने को मिले कि दंगे में इतने मुसलमान मारे गए, तो कट्टरपंथी हिन्दु के मुंह से निकलता है अच्छा, इन्हें और सबक मिलना चाहिए और यदि हिन्दु मारे गए तो कट्टर मुस्लमान अपनी छाती ठोंकता है। अखबारों में धर्म-निरेक्षता वाली खबरों से ही शांति और सामंजस्य बना सकते हैं और इसी माध्यम से एकता का संचार कर सकते हैं।

मेरे साक्षात्कार- भीष्मसाहनी-किताब घर प्रकाशन, पृष्ट-194

\section{3 शोध का उद्देश्य}

1. प्रकाशित समाचार पत्रों में भाषा का इस्तेमाल

2. समाचार पत्र में पेज का चुनाव

3. फोटो का चुनाव

4. क्या खबरें बहुत कम रहती है

5. पाठकों द्वारा प्रतिक्रिया

\section{4 उपकल्पना}

1. स्थानी स्तर से प्रकाशित समाचार पत्रों में धर्म-निरपेक्षता से सम्बंधित खबरों में शुद्ध भाषा का इस्तेमाल नहीं हो रहा है।

2. स्थानी स्तर से प्रकाशित समाचार पत्रों में धर्म-निरपेक्षता से सम्बंधित खबरों के लिए पेज निर्धारित नहीं है।

3. रायपुर से प्रकाशित समाचार पत्रों में धर्म-निरपेक्षता से सम्बंधित खबरों के साथ फोटो के लिए बहुत ही कम स्थान दिया जाता है।

4. स्थानीय अखबारों में धर्म-निरपेक्षता से सम्बंधित खबरें बहुत कम होती है।

5. प्रकाशित समाचार पत्रों में धर्म-निरपेक्षता से सम्बंधित खबरों को लेकर पाठकों की प्रतिक्रिया नकारात्मक है।

\section{5 अध्ययन क्षेत्र एक परिचय}

शोध से सम्बंधित अध्ययन क्षेत्र में रायपुर से प्रकाशित समाचार पत्रों का चुनाव किया गया है। शोधार्थी ने नवभारत, भास्कर, पत्रिका और नई दुनिया खबरों का १५ से २७ अगस्त तक अध्ययन किया है। रायपुर से प्रकाशित समाचार पत्रों में धर्म-निरपेक्षता से सम्बंधित खबरों के लिए पेज निर्धारित नहीं है। इसके अलावा इससे जुड़ी हुई खबरों की गंभीरता को देखते हुए पेज, फोटो और कितनी बड़ी खबरें लेनी है आदि निर्धारित किया जाता है। 


\section{6 शोध प्रविधि}

प्रस्तुत शोध के लिए विश्लेषणात्मक शोध प्रविधि का प्रयोग किया गया है। इस शोध विवि को अन्तर्वस्तु विश्लेषण प्रविधि भी कहते हैं। विश्लेषण प्रविधि किसी सामाजिक शोध प्रविधि है जिसके द्वारा विभिन्न स्रोतों से प्राप्त गुणात्मक सामग्री को इस प्रकार व्यवस्थित और श्रेणीबद्ध किया जाता है कि वह परिणाम सही आ सके। केवल सामग्री का विस्तृत विवरण और वर्गीकरण प्रस्तुत करना प्रयाप्त नहीं है। उनका विश्लेषण करना एक कदम आगे बढ़ना है। पर उच्च कोटी के शोध के लिए अध्ययन में विश्लेषणात्मक शोध जरूरी होता है, जिसका गहना चिंतन जरूरी होता है। इसी लिए शोधार्थी अपने शोध में विश्लेषणात्मक प्रविधि के आधार शोध किया है। इसी विधि के द्वारा शोधार्थी ने १५ अगस्त से २७ अगस्त यानी १७ दिनों तक रायपुर से प्रकाशित चार अखबारों का गहना अध्ययन किया है। धर्म-निपेक्षता से सम्बंधित किस तरह की खबरें किस पेज पर प्रकाशित हुई और कितना स्थान मिला। इसी संदर्भ में किस अखबार के इससे जुड़ी हुई खबरों को कितना महत्व दिए, इसका भी अध्ययन किया गया है। वहीं खबरों के साथ फोटो पर विशेष ध्यान दिया गया कि नहीं, फोटो ली गई है या नहीं। यदि फोटो का स्थान मिला है तो कितना। इस तरह की खबरें छपने के बाद पाठकों के बीच किस तरह की प्रतिक्रिए हैं। आदि का विश्लेषण किया गया है।

\section{7 शोध परिणाम}

पत्रिका

इन १७ दिनों में पत्रिका ने धर्म-निरपेक्षता से जुड़ी हुई सिर्फ ६ खबरों को स्थान दिया जिसमें से तीन स्वयं की स्टोरी प्लॉन खबरें थीं। लेकिन इस खबर से पाठकों पर क्या प्रभाव पड़ रहा कोई भी खबरें प्रकाशित नहीं हुई। दो खबरें प्रथम पेज पर एंकर के रूप में फोटो के साथ लगाई गई, बाकि तीन खबरें और एक फोटो अंदर के पेज संख्या सात और नौ पर स्थान दिया गया।

दैनिक भास्कर

सिर्फ 8 खबरों को स्थान दिया। एक खबर प्रथम पेज और बाकी अंदर के पेज पर। भास्कर ने भी पाठकों पर क्या प्रभाव पड़ रहा कोई भी खबरें प्रकाशित नहीं की।

नई दुनिया और नवभारत

नई दुनिया ने तीन और नवभारत ने सिर्फ दो खबरों को स्थान दिया। इन्होंने भी सिर्फ एक-एक खबर को प्रथम पेज पर और बाकी खबरों को अंदर के पेज पर प्रकाशित किया गया है।

1. रायपुर से प्रकाशित समाचार पत्रों में धर्म-निरपेक्षता से सम्बंधित खबरें छपती है, लेकिन बहुत कम।

2. धर्म-निरपेक्षता से जुड़ी हुई खबरों के लिए सामान्य भाषा का इस्तेमाल किया जाता है और स्थानीय भाषा पर जोर दिया गया है।

3. इस तरह की खबरों के लिए कोई विशेष पेज नहीं है लेकिन धर्म से जुड़ी हुई खबरों के लिए हर समाचार पत्रों ने स्थान निर्धारित या पेज संख्या निर्धारित किया है।

4. धर्म-निरपेक्षता से जुड़ी हुई खबरों के साथ समाचार पत्रों में फोटो भी दिए गए हैं, लेकिन फोटो अक्सर छोटी लगायी गई है।

5. यह दुर्भाग्य है कि जब भी इस तरह की खबरें प्रकाशित होती है उसका पाठकों पर क्या प्रभाव पड़ता है खबरें होनी चाहिए, लेकिन बहुत कम ऐसी खबरें होती हैं जो समाचार पत्र प्रकाशित करते हैं। 
6. प्रथम पेज पर जो खबरें प्रकाशित हुई वह चार से पांच कॉलम की है और अंदर के पेजों पर भी तीन से चार कॉलम मिला है।

\section{8 सारांश, निष्कर्ष एवं सुझाव}

रायपुर से प्रकाशित समाचार पत्रों में धर्म-निरपेक्षता से सम्बंधित खबरों का अध्ययन के लिए शोधार्थी ने विश्लेषणात्मक शोध विधि का प्रयोग किया। १५ अगस्त से २७ तक अगस्त यानी १७ दिनों तक नवभारत, भास्कर, पत्रिका और नई दुनिया समाचार पत्रों का अध्ययन किया। इसके परिणाम निकलकर यह आया कि रायपुर में हर धर्म के लोग सर्वाधिक संख्या में रहते हैं। सभी एक दूसरे से जुड़े हुए है। अक्सर देखने को भी मिलता है कि उनकी खुशियों में अपने धर्म को किनारे कर समाज में एक अच्छा संदेश भी देते हैं। धर्म से जुड़ी हुई खबरों के पेज निर्धारित है, लेकिन धर्म-निरपेक्षा से सम्बंधित खबरों के लिए नहीं। कभी-कभार उचित स्थान भी नहीं मिलता है। फोटो के लिए भी स्थान कम पड़ जाता है। विज्ञापन होने के कारण खबरों से अन्याय तो होता ही है फोटो के लिए भी स्थान न के बराबर है। आस्था या किसी सम्प्रदाय से जुड़ी हुई खबरों के लिए भाषा का अभाव है।

\section{9 सुझाव}

1. धर्म-निरपेक्षता से सम्बंधित खबरों को स्थान मिलना चाहिए।

2. कभी-कभार फोटो भी अपने-आप में सम्पूर्ण खबर होती है उसे उचित स्थान मिलना चाहिए।

3. पाठक हमेशा शिकायत करते हैं कि धर्म-निरपेक्षता से सम्बंधित खबरों को स्थान नहीं मिलता है। यदि मिल भी जाए तो पाठकों के बीच क्या संदेश जाता है इससे जुड़ी खबरें बहत कम रहती है। जब यह एक गंभीर विषय है तो पाठकों के विचार होने चाहिए।

4. इस तरह की खबरें रुचिकर होती है, इसलिए प्रथम या लास्ट पेज पर स्थान मिले तो अच्छा रहेगा।
